\title{
Temperature and electric potential fields of an interface crack in a layered
}

\section{thermoelectric or metal/thermoelectric material}

\author{
Aibing Zhang ${ }^{1 *}$, Baolin Wang ${ }^{2}$ \\ ${ }^{1}$ Piezoelectric Device Laboratory, School of Mechanical Engineering \& Mechanics, \\ Ningbo University, Ningbo, 315211, P.R. China \\ ${ }^{2}$ Institute for Infrastructure Engineering, Western Sydney University, Penrith, NSW \\ 2751, Australia
}

\begin{abstract}
The interface crack problem in a layered thermoelectric or metal/thermoelectric material subjected to thermoelectric loadings is studied based on the nonlinear governing equations and complex variable method. The electric impermeable and heat semi-permeable crack boundary conditions are adopted. Explicit and closed-form solutions of temperature and electric potential on the interface crack surfaces, and crack tip thermoelectric fields are obtained. The influence of crack thickness to length ratio and thermal conductivity of air inside the crack on electric current density and heat flux intensity factors at crack tip is discussed. It is found that electric current density and heat flux intensity factors at the interface crack tip between metal interconnector and thermoelectric material are much bigger than those in homogenous or layered thermoelectric material. This explains why the failure (or microcrack initiation) of a thermoelectric generation/cooler element always appears at the interface between the metal electrode and thermoelectric material.
\end{abstract}

Keywords: Thermoelectric material; Interface crack; Intensity factors

${ }^{*}$ Corresponding author. Tel.: +86 75526032119

E-mail address: aibingzhang.hit@gmail.com (A.B. Zhang). 


\section{Introduction}

The research and development of thermoelectric materials have attracted significant interests recently owing to their capability in converting heat energy into electric energy directly utilizing the Seebeck effect and vice versa [1,2]. Being all solid state and without moving parts, thermoelectric devices can be applied to waste heat recovery [3], refrigeration [4], carbon reduction [5] and solar energy harvesting [6]. Design of thermoelectric intelligent devices is calling for a better understanding of the responses of these materials subjected to applied temperature and electric potential loadings. The influence of the Thomson effect on the performance of a thermoelectric cooler was studied by Huang et al. [7]. Finite element analysis of nonlinear fully coupled thermoelectric materials based on continuum mechanics and the Galerkin method was carried out by Pérez-Aparicio et al. [8]. A general, three-dimensional thermoelectric material model by taking the Joule heating, Fourier's heat conduction, the Thomson effect, the Peltier effect, the convection and radiation heat transfer, and temperature dependent properties of semiconductor materials into account was presented by Wang et al. [9]. A continuum theory for thermoelectric medium following the general framework of continuum mechanics and conforming to basic thermodynamic laws was developed by Liu [10]. Dynamic response characteristics of thermoelectric generator predicted by a 3D heat-electricity coupled model was investigated by Meng et al. [11].

Massive efforts on thermoelectric materials have been directed towards improving their figure of merit and energy conversion efficiency [12-14]. The pioneering works of $[15,16]$ have shown that the energy conversion efficiency of a layered thermoelectric composite can be higher than all its constituents due to the nonlinear distribution of temperature and electric potential inherent in thermoelectric transport. 
This conclusion may point to a new route for high figure of merit thermoelectric materials. The formulas for thermoelectric devices that operate in parallel were derived by Mahan [17]. A rigorous nonlinear asymptotic homogenization theory to analyze the coupled transport of electricity and heat in thermoelectric composite materials was developed by Yang et al. [18]. On the other hand, it is well known that thermoelectric materials are typical brittle solids. Defects/imperfections produced during the manufacturing and/or in-service condition, such as cracks, can cause geometric, temperature and electric potential discontinuities across the crack faces, and thermoelectric fields concentrations at the crack tip, thus contribute to critical crack growth. The crack problem in a thermoelectric medium under thermal-electric loads was considered by Zhang and Wang [19]. The effective material properties of thermoelectric composites with elliptical fibers were studied by Wang [20]. The general solutions for the two-dimensional problem in a cracked thermoelectric materials were derived by Song et al. [21]. Further, an overwhelming majority of thermoelectric devices fracture failure begins at the interface between metal electrode and thermoelectric material, or two different thermoelectric materials. Cui et al. [22] observed that cracks occurred near the interface between $\mathrm{Sn}_{95} \mathrm{Ag}_{5}$ and $\mathrm{Bi}_{2} \mathrm{Te}_{3}$ after thermal stability testing at $192{ }^{\circ} \mathrm{C}$ annealing for $200 \mathrm{~h}$. The interface characterization of nickel contacts to bulk bismuth tellurium selenide was reported by Lyore et al. [23]. $\mathrm{CoSb}_{3}$-based thermoelectric element with insertion of Ti layer by means of spark plasma sintering was obtained by Zhao et al. [24], and they found that the cracks appeared near the interface due to the different thermal expansion coefficient of $\mathrm{CoSb}_{3}$ and Ti. Therefore, the investigation of interface crack problem in layered thermoelectric or metal/thermoelectric materials is very important.

In view of the above literature analysis, the purpose of this paper is to present the 
solutions of temperature and electric potential distribution for an interface crack problem in a layered thermoelectric or metal/thermoelectric heterogeneous material. The influence of the thermal conductivity of air inside the crack on the crack tip fields is also studied based on the finite thickness crack model. The paper is organized as follows. Firstly, the governing equations of thermoelectric material are outlined. Next, solutions of electric and temperature fields of a crack-free and a cracked layered thermoelectric material are obtained in closed-form in Sections 2 and 3, respectively. Some numerical results are given in Section 4. Finally, concluding remarks on interface crack problem are made.

\section{Governing equations}

Consider an infinite thermoelectric material in which all fields are assumed to depend only on the in-plane coordinates $x$ and $y$. In the stationary case when no free electric charge and heat source exists, the fully nonlinearly coupled governing equations for a homogeneous thermoelectric material can be written as [8],

$$
\begin{gathered}
\nabla \cdot \mathbf{j}_{e}=0 \\
\nabla \cdot \mathbf{q}+\mathbf{j}_{e} \cdot \nabla V=0
\end{gathered}
$$

together with

$$
\begin{gathered}
\mathbf{j}_{e}=-\sigma \nabla V-\sigma s \nabla T \\
\mathbf{q}=-\sigma s T \nabla V-\left(\kappa+\sigma s^{2} T\right) \nabla T
\end{gathered}
$$

where $V$ is the electric potential and $T$ is the temperature function; $\mathbf{j}_{e}=\left[j_{e x}, j_{e y}\right]^{T}$ is the electric current density vector, $\mathbf{q}=\left[q_{x}, q_{y}\right]^{T}$ is the heat flux vector; $\sigma, \kappa$ and $s$ are the electric conductivity, thermal conductivity and Seebeck coefficient, respectively. Here, suppose that thermoelectric properties of a single material are independent of temperature and size, i.e., $\sigma, \kappa$ and $s$ are constants. Since energy 
is transported by both electrons and heat, the energy flux vector $\mathbf{j}_{u}=\left[j_{u x}, j_{u y}\right]^{T}$ can be expressed as

$$
\mathbf{j}_{u}=\mathbf{q}+\mathbf{j}_{e} V
$$

In order to facilitate the solution procedure, a new potential function $F$ is introduced, and defined as

$$
F=V+s T
$$

It is clear that the physical meaning of $F$ is the total electric potential function including the Seebeck effect from Eq. (4). Combining Eqs. (2) - (4), the constitutive equations can be rewritten as,

$$
\begin{gathered}
\mathbf{j}_{e}=-\sigma \nabla F \\
\mathbf{j}_{u}=-\sigma F \nabla F-k \nabla T
\end{gathered}
$$

Substituting Eqs. (3) and (5) into Eq. (1), the governing equations can be expressed as,

$$
\begin{gathered}
\nabla^{2} F=0 \\
k \nabla^{2} T+\sigma(\nabla F)^{2}=0
\end{gathered}
$$

where the total electric potential function $F$ is uncoupled from the whole problem and satisfies the Laplace's equation, that is, the total electric potential may be obtained directly form the theory of electrostatics, and then the temperature field can be given with the aid of the known electric field.

\section{Temperature and electric potential fields of a crack-free layered}

\section{thermoelectric material}

Consider a layered thermoelectric composite material consisting of two crack-free homogeneous thermoelectric phases 1 and 2 with the lengths $L_{1}$ and $L_{2}$, $S^{+}$and $S^{-}$are upper $(y>0)$ and lower $(y<0)$ regions, respectively. Assume that the specified electric potentials and temperatures of $\left(V_{10}, T_{10}\right)$ and $\left(V_{20}, T_{20}\right)$ are 
applied at both ends, as shown in Fig. 1 (without taking the interface crack into consideration in this section). It is found that electric current density and energy flux are constant distribution for such one dimension layered thermoelectric material transport $[15,25]$, that is, $j_{e y 1}=j_{e y 2}=j_{e 0}$ and $j_{u y 1}=j_{u y 2}=j_{u 0}$, the subscripts 1 and 2 refer to material 1 and material 2, respectively. Then the governing temperature and electric potential fields can be simplified as follows from Eq. (6),

$$
\frac{d^{2} F_{k}}{d y^{2}}=0, \frac{d^{2} T_{k}}{d y^{2}}=-\frac{j_{e 0}^{2}}{\kappa_{k} \sigma_{k}}
$$

where the subscript $k$ can be changed to 1,2 and refer to material $1(y>0)$, material 2 $(y<0)$, respectively. The solutions of $F_{k}$ and $T_{k}$ have the form,

$$
F_{k}=c_{1 k} y+c_{2 k}, T_{k}=-\frac{j_{e 0}^{2}}{2 \kappa_{k} \sigma_{k}} y^{2}+c_{3 k} y+c_{4 k}
$$

where $c_{1 k}, c_{2 k}, c_{3 k}$ and $c_{4 k}$ are constants to be determined. The electric potential and temperature boundary conditions at both ends can be written as

$$
\begin{aligned}
& V_{1}\left(L_{1}\right)=V_{10}, T_{1}\left(L_{1}\right)=T_{10} \\
& V_{2}\left(-L_{2}\right)=V_{20}, T_{2}\left(-L_{2}\right)=T_{20}
\end{aligned}
$$

Material 1 and material 2 are rigidly bonded together so that

$$
\begin{aligned}
& V_{1}\left(y=0^{+}\right)=V_{2}\left(y=0^{-}\right) \\
& T_{1}\left(y=0^{+}\right)=T_{2}\left(y=0^{-}\right)
\end{aligned}
$$

Combination of (8) with the boundary conditions (9) and $(10)_{2}$ gives 


$$
\begin{aligned}
& c_{11}=-j_{e 0} / \sigma_{1}, c_{12}=-j_{e 0} / \sigma_{2} \\
& c_{21}=F_{10}+\frac{L_{1} j_{e 0}}{\sigma_{1}}, c_{22}=F_{20}-\frac{L_{2} j_{e 0}}{\sigma_{2}} \\
& c_{31}=\kappa_{2} \frac{T_{10}-T_{20}+j_{e 0}^{2} L_{1}^{2} /\left(2 \kappa_{1} \sigma_{1}\right)-j_{e 0}^{2} L_{2}^{2} /\left(2 \kappa_{2} \sigma_{2}\right)}{\kappa_{1} L_{2}+\kappa_{2} L_{1}} \\
& c_{32}=\kappa_{1} \frac{T_{10}-T_{20}+j_{e 0}^{2} L_{1}^{2} /\left(2 \kappa_{1} \sigma_{1}\right)-j_{e 0}^{2} L_{2}^{2} /\left(2 \kappa_{2} \sigma_{2}\right)}{\kappa_{1} L_{2}+\kappa_{2} L_{1}} \\
& c_{41}=c_{42}=\frac{\kappa_{1} L_{2} T_{10}+\kappa_{2} L_{1} T_{20}}{\kappa_{1} L_{2}+\kappa_{2} L_{1}}+\frac{j_{e 0}^{2} L_{1} L_{2}\left(\sigma_{1} L_{2}+\sigma_{2} L_{1}\right)}{2 \sigma_{1} \sigma_{2}\left(\kappa_{1} L_{2}+\kappa_{2} L_{1}\right)}
\end{aligned}
$$

where $F_{10}=V_{10}+s_{1} T_{10}$ and $F_{20}=V_{20}+s_{2} T_{20}$. From the continuity of electric potential at the interface, Eq. (10) 1 can be reduced to ,

$$
A_{2} j_{e 0}^{2}+A_{1} j_{e 0}+A_{0}=0
$$

where

$$
\begin{aligned}
& A_{2}=\frac{\left(s_{2}-s_{1}\right)\left(\sigma_{1} L_{2}+\sigma_{2} L_{1}\right) L_{1} L_{2}}{2 \sigma_{1} \sigma_{2}\left(\kappa_{1} L_{2}+\kappa_{2} L\right)}, A_{1}=\frac{L_{1}}{\sigma_{1}}+\frac{L_{2}}{\sigma_{2}}, \\
& A_{0}=\frac{\left(s_{2}-s_{1}\right)\left(\kappa_{1} L_{2} T_{10}+\kappa_{2} L_{1} T_{20}\right)}{\kappa_{1} L_{2}+\kappa_{2} L}+F_{10}-F_{20}
\end{aligned}
$$

For reasonable material properties and loading conditions, Eq. (12) has two roots, but only one root is physically acceptable. In this paper, the solution of electric current density has the following form,

$$
j_{e 0}=\frac{-A_{1}-\sqrt{A_{1}^{2}-4 A_{0} A_{2}}}{2 A_{2}}
$$

From Eq. (5) 2 , the energy flux $j_{u 0}$ is obtained as,

$$
j_{u 0}=F_{10}+\frac{j_{e 0}^{2} L_{1}}{\sigma_{1}}-\kappa_{1} c_{31}
$$

The heat flux $q_{y k}$ can then be calculated by using Eq. (3). Obviously, heat flux has a nonlinear relations with $y$. Now, the electric potential and temperature fields in a layered thermoelectric heterogeneous material are completely solved. 


\section{Temperature and electric potential fields of an interface crack in a layered thermoelectric material}

\subsection{Sate of the problem}

Consider a layered thermoelectric heterogeneous material containing a through interface crack with its length being $2 a$ and denote by $(x, y)$ the rectangular coordinates system with its origin at the middle point of the crack, as shown in Fig. 1. The layered thermoelectric material is subjected to the specified electric potentials and temperatures of $\left(V_{10}, T_{10}\right)$ and $\left(V_{20}, T_{20}\right)$. If the crack length is very small comparing with the size of thermoelectric material and the location of the crack is far away from the boundary, the following boundary conditions are adopt from the results of the previous section,

$$
\begin{aligned}
& j_{e x 1}=j_{e x 2}=0, j_{e y 1}=j_{e y 2}=j_{e 0} \\
& j_{u x 1}=j_{u x 2}=0, j_{u y 1}=j_{u y 2}=j_{u 0}
\end{aligned}, \text { as } \sqrt{x^{2}+y^{2}} \rightarrow \infty
$$

In engineering practices, flaws in thermoelectric material are not like cleavage cracks of zero gap width, but rather like notches with a finite thickness, and crack is always filled with air which is a good electrical insulator. The electric conductivity of air is almost $10^{16} \sim 10^{18}$ orders of magnitude less than that of thermoelectric material, and can be neglected. In addition, electrical insulated crack model has been widely used in other functional materials for the interface crack problem [26, 27]. Therefore, the boundary condition of electric current density at the crack surfaces can be supposed to be impermeable, i.e.,

$$
\begin{array}{ll}
j_{e y 1}=j_{e y 2}=0, & |x| \leq a, y=0 \\
j_{e y 1}=j_{e y 2}, & |x| \geq a, y=0 \\
V_{1}=V_{2}, & |x| \geq a, y=0
\end{array}
$$

In order to obtain high energy conversion efficiency, the thermoelectric material needs to have high Seebeck coefficient, high electric conductivity, and low heat conductivity. 
Generally, the heat conductivity of thermoelectric material is almost $10-100$ times more than that of air. However, the heat flux through the crack should not be zero since the crack opening displacement $\delta(x)$ is always very small [28]. From Eqs. (3) and (16) $)_{1}$, we have $j_{u y 1}=j_{u y 2}=q_{y}^{c}$ at the crack surfaces, where $q_{y}^{c}$ is the heat flux inside the crack. Along the $y$-axis the normal components of the energy flux boundary condition can be written in terms of the temperature jumps $\Delta T$ as [29],

$$
\begin{array}{ll}
j_{u y 1}=j_{u y 2}=j_{u y}^{c}=q_{y}^{c}=-\kappa_{c} \frac{\Delta T}{2 \delta(x)}, & |x| \leq a, y=0 \\
j_{u y 1}=j_{u y 2}, T_{1}=T_{2}, & |x| \geq a, y=0
\end{array}
$$

where $\kappa_{c}$ stands for the thermal conductivity of the air inside the crack.

\subsection{Electric field}

The solution of Eq. $(6)_{1}$ is:

$$
F_{k}=\operatorname{Re}\left[f_{k}(z)\right], z=x+i y
$$

where $f_{k}(z)$ is the unknown potential function to be determined, and "Re" stands for the real part of a complex number. Inserting Eq. (18) in Eq. (5) $)_{1}$, we obtain:

$$
j_{\text {exk }}=-\sigma_{k} \operatorname{Re}\left[f_{k}^{\prime}(z)\right], j_{\text {eyk }}=\sigma_{k} \operatorname{Im}\left[f_{k}^{\prime}(z)\right]
$$

where "Im" stands for the imaginary part of a complex number.

Substituting Eq. (18) into Eq. (6) 2 yields:

$$
\nabla^{2} T_{k}=-\frac{\sigma_{k}}{\kappa_{k}}\left(\nabla F_{k}\right)^{2}=-\frac{\sigma_{k}}{\kappa_{k}} f_{k}^{\prime}(z) \overline{f_{k}^{\prime}(z)}
$$

For the problem considered here it is convenient to represent the temperature field as the sum of two functions:

$$
T_{k}=T_{1 k}+T_{2 k}
$$

where $T_{1 k}$ and $T_{2 k}$ are satisfied the following equation, respectively, 


$$
\nabla^{2} T_{1 k}=-\frac{\sigma_{k}}{\kappa_{k}} f_{k}^{\prime}(z) \overline{f_{k}^{\prime}(z)}, \nabla^{2} T_{2 k}=0
$$

It is easy to find from Eq. (22) that

$$
T_{1 k}=-\frac{\sigma_{k}}{4 \kappa_{k}} f_{k}(z) \overline{f_{k}(z)}, T_{2 k}=\operatorname{Re}\left[g_{k}(z)\right]
$$

where $g_{k}(z)$ is arbitrary analytic function. From Eqs. (5), (18) and (21)-(23), the energy flux can then be obtained as

$$
\begin{aligned}
& j_{u x k}=-\operatorname{Re}\left[\frac{\sigma_{k}}{2} f_{k}(z) f_{k}^{\prime}(z)+\kappa_{k} g_{k}{ }^{\prime}(z)\right] \\
& j_{u y k}=\operatorname{Im}\left[\frac{\sigma_{k}}{2} f_{k}(z) f_{k}^{\prime}(z)+\kappa_{k} g_{k}{ }^{\prime}(z)\right]
\end{aligned}
$$

By using Eqs. (16) 2,3 , the following sectionally holomorphic functions $\phi^{\prime}(z)$ and $\varphi^{\prime}(z)$ are defined in the forms,

$$
\begin{gathered}
\phi^{\prime}(z)=\sigma_{1} f_{1}^{\prime}(z)+\sigma_{2} \bar{f}_{2}^{\prime}(z), \quad z \in S^{+} \\
\phi^{\prime}(z)=\sigma_{1} \bar{f}_{1}^{\prime}(z)+\sigma_{2} f_{2}^{\prime}(z), \quad z \in S^{-} \\
\varphi^{\prime}(z)=f_{1}^{\prime}(z)-\bar{f}_{2}^{\prime}(z), \quad z \in S^{+} \\
\varphi^{\prime}(z)=-\bar{f}_{1}^{\prime}(z)+f_{2}^{\prime}(z), \quad z \in S^{-}
\end{gathered}
$$

The potential functions $\phi^{\prime}(z)$ and $\varphi^{\prime}(z)$ have the form of

$$
\begin{aligned}
& \phi^{\prime}(z)=c_{1}^{\infty}+\phi_{0}^{\prime}(z) \\
& \varphi^{\prime}(z)=c_{2}^{\infty}+\varphi_{0}^{\prime}(z)
\end{aligned}
$$

where $c_{1}^{\infty}$ and $c_{2}^{\infty}$ are two constants to be determined, $\phi_{0}^{\prime}(z)$ and $\varphi_{0}^{\prime}(z)$ are two holomorphic function in the z-plane except on the crack faces, and $\phi_{0}^{\prime}(\infty)=0$ and $\varphi_{0}^{\prime}(\infty)=0$. Combining electric current density boundary conditions $(15)_{1}$, and substituting Eqs. (25)-(27) into Eq. (19), then taking the limiting $z \rightarrow \infty$ yields 


$$
c_{1}^{\infty}=0, c_{2}^{\infty}=i j_{e 0} \frac{\sigma_{1}+\sigma_{2}}{\sigma_{1} \sigma_{2}}
$$

Making use of the boundary condition (16) $)_{1}$, gives

$$
\begin{array}{ll}
\phi_{0}^{\prime}(x)^{+}-\phi_{0}^{\prime}(x)^{-}=0, & |x| \leq a, y=0 \\
\varphi_{0}^{\prime}(x)^{+}+\varphi_{0}^{\prime}(x)^{-}=-i j_{e 0} \frac{\sigma_{1}+\sigma_{2}}{\sigma_{1} \sigma_{2}}, & |x| \leq a, y=0
\end{array}
$$

Based on the Muskhelishvili theorem [30], the solution of boundary value problem (29) can be obtained as

$$
\begin{gathered}
\phi_{0}^{\prime}(z)=0 \\
\varphi_{0}^{\prime}(z)=-\frac{X(z)}{2 \pi i} \int_{-a}^{a} \frac{X^{-1}(x)^{+}}{x-z} \frac{2 i j_{e 0}\left(\sigma_{1}+\sigma_{2}\right)}{\sigma_{1} \sigma_{2}} d x
\end{gathered}
$$

where $X(z)=\frac{1}{\sqrt{z^{2}-a^{2}}}$.

The integration of (31) with respect to $x$ leads to

$$
\varphi_{0}^{\prime}(z)=-\frac{i j_{e 0}\left(\sigma_{1}+\sigma_{2}\right)}{\sigma_{1} \sigma_{2}}\left[1-\frac{z}{\sqrt{z^{2}-a^{2}}}\right]
$$

Finally, we have

$$
\begin{gathered}
\phi^{\prime}(z)=0 \\
\varphi^{\prime}(z)=\frac{i j_{e 0}\left(\sigma_{1}+\sigma_{2}\right)}{\sigma_{1} \sigma_{2}} \frac{z}{\sqrt{z^{2}-a^{2}}}
\end{gathered}
$$

\subsection{Temperature field}

By using Eq. $(17)_{2}$ the sectionally holomorphic functions $\Theta^{\prime}(z)$ and $\Phi^{\prime}(z)$ are defined in the forms,

$$
\begin{aligned}
& \Theta^{\prime}(z)=\kappa_{1} g_{1}^{\prime}(z)+\kappa_{2} \bar{g}_{2}^{\prime}(z), \quad z \in S^{+} \\
& \Theta(z)=\kappa_{1} \bar{g}_{1}^{\prime}(z)+\kappa_{2} g_{2}{ }^{\prime}(z), \quad z \in S^{-}
\end{aligned}
$$




$$
\begin{aligned}
& \Phi^{\prime}(z)=g_{1}^{\prime}(z)-g_{2}{ }^{\prime}(z)-\frac{\sigma_{1}}{2 \kappa_{1}} \bar{\omega}_{1}(z)+\frac{\sigma_{2}}{2 \kappa_{2}} \omega_{2}(z), \quad z \in S^{+} \\
& \Phi^{\prime}(z)=-g_{1}^{\prime}(z)+g_{2}^{\prime}(z)+\frac{\sigma_{1}}{2 \kappa_{1}} \omega_{1}(z)-\frac{\sigma_{2}}{2 \kappa_{2}} \bar{\omega}_{2}(z), \quad z \in S^{-}
\end{aligned}
$$

where

$$
\omega_{k}(z)=f_{k}^{\prime}(\bar{z}) \overline{f_{k}}(z)
$$

Eq. (24) shows that $\Theta^{\prime}(z)$ and $\Phi^{\prime}(z)$ should have the same form as $f_{k}(z) f_{k}^{\prime}(z)$ in order to satisfy the single valued conditions of electric potential and temperature. Thus, by observing Eqs. (25)-(27), (33) and (34) one can express $\Theta^{\prime}(z)$ and $\Phi^{\prime}(z)$ in the form

$$
\begin{aligned}
& \Theta^{\prime}(z)=c_{3}^{\infty} z+c_{4}^{\infty}+\Theta_{0}^{\prime}(z) \\
& \Phi^{\prime}(z)=c_{5}^{\infty} z+c_{6}^{\infty}+\Phi_{0}^{\prime}(z)
\end{aligned}
$$

where $\Theta_{0}^{\prime}(z)$ and $\Phi_{0}^{\prime}(z)$ stand for two holomorpic functions, and $\Theta_{0}^{\prime}(\infty)=0$ and $\Phi_{0}^{\prime}(\infty)=0, c_{3}^{\infty}, c_{4}^{\infty}, c_{5}^{\infty}$ and $c_{6}^{\infty}$ are four constants to be determined.

Substituting Eqs. (35)-(38) into Eq. (24), and then taking the limit $z \rightarrow \infty$ yields

$$
\begin{aligned}
& c_{3}^{\infty}=\frac{j_{e 0}^{2}\left(\sigma_{1}+\sigma_{2}\right)}{2 \sigma_{1} \sigma_{2}}, c_{4}^{\infty}=0 \\
& c_{5}^{\infty}=0, c_{6}^{\infty}=\frac{i j_{u 0}\left(\kappa_{1}+\kappa_{2}\right)}{\kappa_{1} \kappa_{2}}
\end{aligned}
$$

Eqs. $(17)_{1}$ can be changed to the following equations for energy flux boundary condition by using Eqs. (35) and (36),

$$
\begin{array}{ll}
\Theta_{0}{ }^{\prime}(x)^{+}-\Theta_{0}{ }^{\prime}(x)^{-}=0, & |x| \leq a, y=0 \\
\Phi_{0}{ }^{\prime}(x)^{+}+\Phi_{0}{ }^{\prime}(x)^{-}=-2 i\left(j_{u 0}-j_{u y}^{c}\right) \frac{\kappa_{1}+\kappa_{2}}{\kappa_{1} \kappa_{2}}, & |x| \leq a, y=0
\end{array}
$$

According to Muskhelishvili [30], the solutions of Eq. (40) are given by 


$$
\begin{gathered}
\Theta_{0}{ }^{\prime}(z)=0 \\
\Phi_{0}{ }^{\prime}(z)=-\frac{X(z)}{2 \pi i} \int_{-a}^{a} \frac{X^{-1}(x)^{+}}{x-z} \frac{2 i\left(j_{u 0}-j_{u y}^{c}\right)\left(\kappa_{1}+\kappa_{2}\right)}{\kappa_{1} \kappa_{2}} d x
\end{gathered}
$$

As a result, Eq. (42) becomes,

$$
\Phi_{0}^{\prime}(z)=-i\left(j_{u 0}-j_{u y}^{c}\right) \frac{\kappa_{1}+\kappa_{2}}{\kappa_{1} \kappa_{2}}\left(1-\frac{z}{\sqrt{z^{2}-a^{2}}}\right)
$$

Finally, Eqs. (38) becomes,

$$
\begin{gathered}
\Theta^{\prime}(z)=\frac{j_{e 0}^{2}\left(\sigma_{1}+\sigma_{2}\right)}{2 \sigma_{1} \sigma_{2}} z \\
\Phi^{\prime}(z)=i j_{u y}^{c} \frac{\kappa_{1}+\kappa_{2}}{\kappa_{1} \kappa_{2}}+i\left(j_{u 0}-j_{u y}^{c}\right) \frac{\kappa_{1}+\kappa_{2}}{\kappa_{1} \kappa_{2}}\left(\frac{z}{\sqrt{z^{2}-a^{2}}}\right)
\end{gathered}
$$

If we consider a special case that the thermoelectric material contains a thermally insulated crack and is only loaded by a remote uniform applied heat flux $q_{0}$ (i.e., $j_{e 0}=0$ and $\left.j_{u 0}=q_{0}\right)$, the solution of temperature potential $\Phi^{\prime}(z)$ has the following form

$$
\Phi^{\prime}(z)=i j_{u 0} \frac{\kappa_{1}+\kappa_{2}}{\kappa_{1} \kappa_{2}}\left(\frac{z}{\sqrt{z^{2}-a^{2}}}\right)
$$

This result agrees with Eq. (20) in [31] for the thermoelastaic interface crack problem.

\subsection{Intensity factors}

The electric current density $j_{e y k}$ and energy flux $j_{u y k}$ are obtained from Eqs. (19), (24), (33), (34), (44) and (45),

$$
j_{e y k}=\operatorname{Re}\left[j_{e 0} \frac{z}{\sqrt{z^{2}-a^{2}}}\right]
$$




$$
\begin{aligned}
j_{u y k}=\operatorname{Im}[ & -\frac{j_{e 0}^{2}}{2 \sigma_{k}} z+i j_{u y}^{c}+i\left(j_{u 0}-j_{u y}^{c}\right) \frac{z}{\sqrt{z^{2}-a^{2}}}+\frac{\left(\sigma_{1}+\sigma_{2}\right) \kappa_{k} j_{e 0}^{2}}{2\left(\kappa_{1}+\kappa_{2}\right) \sigma_{1} \sigma_{2}} z \\
& \left. \pm \frac{\left(\kappa_{2} \sigma_{2}-\kappa_{1} \sigma_{1}\right) j_{e 0}^{2}}{2\left(\kappa_{1}+\kappa_{2}\right) \sigma_{1} \sigma_{2}} \frac{\bar{z} \sqrt{z^{2}-a^{2}}}{\sqrt{-2}-a^{2}}\right]
\end{aligned}
$$

where "+" for $j_{u y 1}$, and "- " for $j_{u y 2}$.

The temperature field $T_{k}$ and electric potential $V_{k}$ are expressed as,

$$
\begin{aligned}
& T_{k}= \operatorname{Re}\left[i \frac{j_{u y}^{c}}{\kappa_{k}} z+i \frac{\left(j_{u 0}-j_{u y}^{c}\right)}{\kappa_{k}} \sqrt{z^{2}-a^{2}}+\frac{\left(\sigma_{1}+\sigma_{2}\right) j_{e 0}^{2}}{4\left(\kappa_{1}+\kappa_{2}\right) \sigma_{1} \sigma_{2}} z^{2}\right] \\
&-\frac{\left(\sigma_{1}+\sigma_{2}\right) j_{e 0}^{2}}{4\left(\kappa_{1}+\kappa_{2}\right) \sigma_{1} \sigma_{2}} \sqrt{z^{2}-a^{2}} \sqrt{z^{2}-a^{2}}+d_{k} \\
& V_{k}=\operatorname{Re}\left[i\left(\frac{j_{e 0}}{\sigma_{k}}-s_{k} \frac{j_{u 0}-j_{u y}^{c}}{\kappa_{k}}\right) \sqrt{z^{2}-a^{2}}-i \frac{s_{k} j_{u y}^{c}}{\kappa_{k}} z-\frac{\left(\sigma_{1}+\sigma_{2}\right) s_{k} j_{e 0}^{2}}{4\left(\kappa_{1}+\kappa_{2}\right) \sigma_{1} \sigma_{2}} z^{2}\right] \\
&+ \frac{\left(\sigma_{1}+\sigma_{2}\right) s_{k} j_{e 0}^{2}}{4\left(\kappa_{1}+\kappa_{2}\right) \sigma_{1} \sigma_{2}} \sqrt{z^{2}-a^{2}} \sqrt{\frac{-2}{z}-a^{2}}-s_{k} d_{k}
\end{aligned}
$$

where $d_{k}$ is an integral constant to be determined. To satisfy the electric potential boundary condition Eq. (16) 3 at the interface, we have

$$
d_{1}=d_{2}=-\frac{\left(\sigma_{1}+\sigma_{2}\right) a^{2} j_{e 0}^{2}}{4\left(\kappa_{1}+\kappa_{2}\right) \sigma_{1} \sigma_{2}}
$$

In this paper, the crack with an elliptic opening displacement is considered such that $[28,32]$

$$
\delta(x)=\frac{\delta_{0}}{a} \sqrt{a^{2}-x^{2}}
$$

where $\delta_{0}$ is crack half-thickness at $x=0$. It is noted that the crack opening displacement $\delta(x)$ is sufficiently small, and the gradient of elliptic opening along the crack surfaces is also small expect near the tip of the crack. The temperature jump across the crack can be obtained as 


$$
\Delta T=T_{1}^{+}-T_{2}^{-}=-\left(j_{u 0}-j_{u y}^{c}\right) \frac{\left(\kappa_{1}+\kappa_{2}\right)}{\kappa_{1} \kappa_{2}} \sqrt{a^{2}-x^{2}}
$$

From Eq. $(17)_{1},(52)$ and (53), it follows that the energy flux inside the crack is

$$
j_{u y}^{c}=\frac{j_{u 0}}{1+\kappa_{1} \kappa_{2}\left(\delta b_{b} a\right) /\left[\left(\kappa++_{1} \kappa\right) \aleph_{c}\right]}
$$

The heat flux $q_{y k}$ also can be obtained by Eqs. (3), (47), (48) and (50). It is found that the electric current density, energy flux and heat flux at the crack tip exhibit traditional inverse square-root singularity. Defining the electric current density, heat flux and energy flux intensity factors $K_{e}, K_{q}$ and $K_{u}$ in the conventional manner, we get that

$$
\begin{aligned}
& K_{e}=\lim _{z \rightarrow a} \sqrt{2 \pi(z-a)} j_{e y k}=j_{e 0} \sqrt{\pi a} \\
& K_{q}=K_{u}=\lim _{z \rightarrow a} \sqrt{2 \pi(z-a)} j_{u y k}=\left(j_{u 0}-j_{u y}^{c}\right) \sqrt{\pi a}
\end{aligned}
$$

It can be found that electric current density intensity factor $K_{e}$ does not depend on the crack-face heat boundary condition assumption from Eq. $(55)_{1}$, the main reason is that the total electric potential function and electric current density are uncoupled from the temperature field and only depended on the applied electric loads ( Eqs. (5) 1 and $\left.(6)_{1}\right)$. In addition, the electric impermeable crack boundary condition is adopted, and this assumption ignores the existence of electric current density in the crack. The similar results are obtained for the crack problem in a homogenous thermoelectric material by Zhang and Wang when the heat conductivity of air inside the crack is neglected [19]. If the material 1 is selected as interconnector with constant properties due to weak thermoelectric effect, such as copper, then the above solutions can be applied directly to study the interface crack problem between the metal electrode and thermoelectric material.

\section{Numerical results and discussion}


The paper establishes the closed-form solution for temperature and electric potential fields near the interface crack which is considerably smaller than the size of bulk materials. The practical values of intensity factors built can be calculated exactly, which may be used for evaluating the reliability of the layered thermoelectric or metal/thermoelectric material. In this section, the influence of thermoelectric loadings and crack thickness to length ratio on the distribution of electric potential and temperature on the crack surfaces, and crack tip fields is investigated. The layered thermoelectric material is assumed to consist of $\mathrm{Ag}\left(\mathrm{Pb}_{1-\mathrm{y}} \mathrm{Sn}_{\mathrm{y}}\right)_{\mathrm{m}} \mathrm{SbTe}_{2+\mathrm{m}}$ as phase 1 and $\mathrm{Bi}_{2} \mathrm{Te}_{3}$ as phase 2 , and the layered metal/thermoelectric material consisting of copper and $\mathrm{Bi}_{2} \mathrm{Te}_{3}$, respectively. Their thermoelectric properties are listed in Table $1[9,15]$. The problem of a layered composite material of length $L=L_{1}+L_{2}=0.02 \mathrm{~m}$ containing an interface crack of length $2 a(a=0.05 L)$ subjected to temperature and electric potential fields $T_{10}=400 \mathrm{~K}, T_{20}=300 \mathrm{~K}, V_{10}=0 \mathrm{~V}$ and $V_{20}=0.01 \mathrm{~V}$ at its boundary is considered in the following numerical analysis. In this case, $q_{y 1}\left(L_{1}\right)>q_{y 2}\left(-L_{2}\right)$ and $j_{e 0}>0$, the layered thermoelectric material converts heat into electric energy (power generation).

Numerical results of temperature and electric potential of a layered copper $/ \mathrm{Bi}_{2} \mathrm{Te}_{3}$ material on the interface crack surfaces $\left(x \leq|a|, y \rightarrow 0^{ \pm}\right)$and the crack extended line $x \geq|a|, y=0$ for the coordinate $x$ and volume fraction are plotted in Figs. 2 and 3. It can be found that the discontinuity values of temperature and electric potential across the heat insulation crack (i.e., $\kappa_{c}=0$ ) are larger than that obtained by considering the heat conductivity of air inside the interface crack, and these values of discontinuity increase with the increasing of volume fraction of copper. The drops of temperature and electric potential on the upper crack surface are very small for the cases of $L_{1} / L=0.25$ and $L_{1} / L=0.5$ due to the super high thermal and electrical conductivity 
of copper. Further, temperature on the lower crack surface is higher than the applied temperature $T_{10}=400 \mathrm{~K}$ on the material boundary since the nonlinear thermoelectric coupling effect. In Figs. 4 and 5 the temperature and electric potential on the crack line are plotted vs. $x$ for the layered $\mathrm{Ag}\left(\mathrm{Pb}_{1-\mathrm{y}} \mathrm{Sn}_{\mathrm{y}}\right)_{\mathrm{m}} \mathrm{SbTe}_{2+\mathrm{m}} / \mathrm{Bi}_{2} \mathrm{Te}_{3} \quad\left(L_{1} / L=0.5\right)$ thermoelectric heterogeneous material. It is clear that the temperature and electric potential are typical elliptic discontinuous across the crack surfaces. However, the thermoelectric fields on the upper and lower crack surfaces are not distributed symmetrically due to the different thermal and electrical conductivity of the two materials. Again, it can be seen that the effect of thermal physical property of air inside the interface crack on the temperature field is very large. In particular, the maximum temperature jump across the crack is $14.7 \mathrm{~K}$ for the heat insulation crack model; while $\Delta T_{\max }=2.6 \mathrm{~K}$ when the heat semi-permeable boundary condition is used.

Figs. 6 and 7 show that normalized crack tip electric current density intensity factor $K_{e} / K_{e 0}$ and heat flux intensity factor $K_{q} / K_{q 0}$ with the different volume fraction $L_{1} / L$, where $K_{e 0}$ and $K_{q 0}$ are the corresponding values of intensity factors of layered thermoelectric material $\mathrm{Ag}\left(\mathrm{Pb}_{1-\mathrm{y}} \mathrm{Sn}_{\mathrm{y}}\right)_{\mathrm{m}} \mathrm{SbTe}_{2+\mathrm{m}} / \mathrm{Bi}_{2} \mathrm{Te}_{3}\left(L_{1} / L=0.25\right)$, respectively. It is found that $K_{e} / K_{e 0}$ and $K_{q} / K_{q 0}$ of layered thermoelectric material vary only slightly with the volume fraction, but for copper/ $\mathrm{Bi}_{2} \mathrm{Te}_{3}$ heterogeneous medium, $K_{e} / K_{e 0}$ and $K_{q} / K_{q 0}$ obviously increase markedly as $L_{1} / L$ is increased, especially for the heat insulation crack model. The main reason may be that the large mismatch between the thermoelectric properties of metal interconnector and thermoelectric material leads to serious electric current density and heat flux concentrating at the interfacial adhesion. This explains why the failure (or microcrack initiation) of a thermoelectric generation/cooler element always appears at 
the interface between the metal electrode and thermoelectric material [22-24]. The dependence of crack tip heat flux intensity factor $K_{q} / K_{q 0}$ on the crack thickness to length ratio $\delta_{0} / a$ is plotted in Fig. 8, here $K_{q 0}$ is the value of $K_{q}$ for the fully impermeable crack assumption. It can be seen that the crack thickness is shown to have a major effect on the crack tip temperature field, and $K_{q} / K_{q 0}$ at the interface crack tip increases monotonously with the increasing value of $\delta_{0} / a$. The idealization of thermal fully permeable crack model is only reasonable when $\delta_{0} / a$ is zero such that the upper and lower crack faces are in contact. Generally, flaw always has a finite thickness, and the high energy conversion efficiency requires thermoelectric material with a low thermal conductivity. If thermal insulated crack assumption is used to calculated the crack tip heat flux intensity factor $K_{q}$, the errors are greater than $23.2 \%$ and $42 \%$ for the cooper $/ \mathrm{Bi}_{2} \mathrm{Te}_{3}$ and $\mathrm{Ag}\left(\mathrm{Pb}_{1-\mathrm{y}} \mathrm{Sn}_{\mathrm{y}}\right)_{\mathrm{m}} \mathrm{SbTe}_{2+\mathrm{m}} / \mathrm{Bi}_{2} \mathrm{Te}_{3}$ heterogeneous material with a crack aspect ratio of 0.05 , respectively. Therefore, the influence of thermal conductivity of air inside the crack and finite thickness of a crack must be assessed.

\section{Conclusion}

A theoretical investigation has been performed on the interface crack problem of layered thermoelectric or metal/thermoelectric heterogeneous material based on the complex variable technique. Explicit and closed-form solutions are derived both in the material and inside the interface crack. The effect of thermal conductivity of air filled in crack and crack thickness to length ratio on the crack tip fields is studied. The numerical results are compared with the ideal thermal impermeable crack solutions. It is found that the heat conductivity of air inside the crack cannot be neglected, and the influence of finite thickness of a crack should be considered. Large mismatch of 
material properties at interface bonding between metal interconnector and thermoelectric material may be the main reason for the failure (or microcrack initiation) of a thermoelectric generation/cooler element.

Acknowledgements: The research was supported by the National Science Foundation of China (NSFC) (Project Nos. 11402063, 11172081 and 11372086), China Postdoctoral Science Foundation (Project No. 2014M550185) and the Natural Science Foundation of Guangdong Province of China (2014A030313696).

\section{References}

[1] A.J. Minnich, M.S. Dresselhaus, Z.f. Ren, G. Chen, Bulk nanostructured thermoelectric materials: current research and future prospects, Energ. Environ. Sci. 2 (2009) 466-479.

[2] G.J. Snyder, E.S. Toberer, Complex thermoelectric materials, Nat. Mater. 7, (2008) $105-114$

[3] D. Narducci, Do we really need high thermoelectric figures of merit? A critical appraisal to the power conversion efficiency of thermoelectric materials, Appl. Phys. Lett. 99 (2011) 102104-1-102104-3.

[4] T.M. Tritt, M.A. Subramanian, Thermoelectric materials, phenomena, and applications: a bird's eye view, MRS Bullet. 31 (2006) 188-194.

[5] L.E. Bell, Cooling, heating, generating power, and recovering waste heat with thermoelectric systems, Science 321 (2008) 1457-1461.

[6] D. Kraemer, B. Poudel, H.P. Feng, J.C. Caylor, B.Yu, X. Yan, Y. Ma, X.W. Wang, D.Z. Wang, A. Muto, K. Mcenaney, M. Chiesa, Z.F. Ren, G. Chen, High-performance flat-panel solar thermoelectric generators with high thermal 
concentration, Nat. Mater. 10 (2011) 532-538.

[7] M.J. Huang, R.H. Yen, A.B. Wang, The influence of the Thomson effect on the performance of a thermoelectric cooler, Int. J. Heat Mass Tran. 48 (2005) 413-418.

[8] J.L. Pérez-Aparicio, R.L. Taylor, D. Gavela, Finite element analysis of nonlinear fully coupled thermoelectric materials, Comput. Mech. 40 (2007) 35-45.

[9] X.D. Wang, Y.X. Huang, C.H. Cheng, C.T. Lin, C.H. Kang, A three-dimensional numerical modeling of thermoelectric device with consideration of coupling of temperature field and electric potential field, Energy 47 (2012) 488-497.

[10] L.P. Liu, A continuum theory of thermoelectric bodies and effective properties of thermoelectric composites, Int. J. Eng. Sci. 55 (2012) 35-53.

[11] J.H. Meng, X.X. Zhang, X.D. Wang, Dynamic response characteristics of thermoelectric generator predicted by a three-dimensional heat-electricity couplied model, J. Power Sources 245 (2014) 262-269.

[12] J.H. Bahk, Z.X. Bian, M. Zebarjadi, J.M.O. Zide, H. Lu, D.Y. Xu, P. Feser, G.H. Zeng, A. Majumdar, A.C. Gossard, A. Shakouri, J.E. Bowers, Thermoelectric figure of merit of $\left(\operatorname{In}_{0.53} \mathrm{Ga}_{0.47} \mathrm{Ass}\right)_{0.8}\left(\operatorname{In}_{0.52} \mathrm{Al}_{0.48} \mathrm{As}\right)_{0.2} \mathrm{III}-\mathrm{V}$ semiconductor alloys, Phys. Rev. B 81 (2010) 235209-1-235209-6.

[13] G.D. Mahan, J.O. Sofo, The best thermoelectric, Appl. Phys. Sci. 93 (1996) 7436-7439.

[14] A.U. Khan, N. Vlachos, T. Kyratsi, High thermoelectric figure of merit of $\mathrm{Mg}_{2} \mathrm{Si}_{0.55} \mathrm{Ge}_{0.05}$ materils doped with Bi and Sb, Csirpta Mater. 69 (2013) 606-609.

[15] Y. Yang, S.H. Xie, F.Y. Ma, J.Y. Li, On the effective thermoelectric properties of layered heterogeneous medium, J. Appl. Phys. 111 (2012) 013510-1-013510-7.

[16] Y. Yang, F.Y. Ma, C.H. Lei, Y.Y. Liu, J.Y. Li, Is thermoelectric conversion 
efficiency of a composite bounded by its constituents? Appl. Phys. Lett. 102 (2013) 053905-1-053905-4.

[17] G.D. Mahan, Parallel thermoelectrics, Phys. Rev. B 87 (2013) 045415-1-045415-3.

[18] Y. Yang, F.Y. Ma, C.H. Lei, Y.Y. Liu, J.Y. Li, Nonlinear asymptotic homogenization and the effective behavior of layered thermoelectric composites, J. Mech. Phys. Solids 61 (2013) 1768-1783.

[19] A.B.Zhang, B.L. Wang, Crack tip field in thermoelectric media, Theor. Appl. Fract. Mech. 66 (2013) 33-36.

[20] Y.Z. Wang, Effective material properties of thermoelectric composites with elliptical fibers, App. Phys. A 119 (2015) 1081-1085.

[21] H.P. Song, C.F. Gao, J.Y. Li, Two-dimensional problem of a crack in thermoelectric materials, J. Thermal Stresses 38 (2015) 325-337.

[22] J.L. Cui, X.B. Zhao, W. M. Zhao, Y.P. Lu, Preparation, thermoelectric properties and interface analysis of n-type graded material $\mathrm{FeSi}_{2} / \mathrm{Bi}_{2} \mathrm{Te}_{3}$, Mater. Sci. Eng. B 94 (2002) 223-228.

[23] O.D. Lyore, T.H. Lee, R.P. Gupta, J.B. White, H.N. Alshareef, M.J. Kim, B.E. Gnade, Interface characterization of nickel contacts to bulk bismuth tellurium selenide, Surf. Interface Anal. 41 (2009) 440-444.

[24] D.G. Zhao, H.R. Geng, X.Y. Teng, Fabricaiton and reliability evaluation of $\mathrm{CoSb}_{3} / \mathrm{W}-\mathrm{Cu}$ thermoelectric element, J. Alloy. Compd. 517 (2012) 198-203.

[25] F. Hao, D.N. Fang, J.Y. Li, Thermoelectric transport in heterogeneous medium: the role of thermal boundary resistance, Eur. Phys. J. Appl. Phys. 58 (2012) 30901-1-30901-6.

[26] Y.E. Park, Crack extension force in a piezoelectric material. J. Appl. Mech. 
57(1990) 647-653.

[27] P. Ma, W.J. Feng, R.K.L. Su, An electrically impermeable and magnetically permeable interface crack with a contact zone in a magnetoelectroelastic bimaterial under uniform magnetoelectromechanical loads. Eur. J. Mech. A/Solids 32(2012) 41-51.

[28] B.L. Wang, Y.W. Mai, Impermeable crack and permeable crack assumptions, which one is more realistic? J. Appl. Mech. 71 (2004) 575-578.

[29] A. Ricoeur, M. Kuna, The thermoelectromechanical J-integral and the thermal permeability of cracks, Key Eng. Mat. 385-387 (2008) 569-572.

[30] N.I. Muskhelishvili, Some basic problems of the mathematical theory of elasticity, Noordhoff, Leiden, 1975.

[31] K.Y. Lee, S.J. Park, Thermal stress intensity factors for partially insulated interface crack under uniform heat flow. Eng. Fract. Mech. 50 (1995) 475-482.

[32] B.L. Wang, Y.W. Mai, Applicability of the crack-free electromagnetic boundary conditions for fracture of magnetoelectroelastic materials. Int. J. Solids Struct. 44(2007) 387-398. 


\section{List of figure captions}

Figure 1. A layered thermoelectric material containing an interface crack of finite thickness

Figure 2. The temperature field on the crack faces and the crack extended line for a layered metal/thermoelectric material

Figure 3. The electric potential field on the crack faces and the crack extended line for a layered metal/thermoelectric material

Figure 4. The temperature field on the crack faces and the crack extended line for a layered thermoelectric material

Figure 5. The electric potential field on the crack faces and the crack extended line for a layered thermoelectric material

Figure 6. Variation of normalized electric current density intensity factor with volume fraction

Figure 7. Variation of normalized heat flux intensity factor with volume fraction

Figure 8. Variation of normalized heat flux intensity factor with crack thickness to length ratio 
List of table caption

Table 1 Material properties 


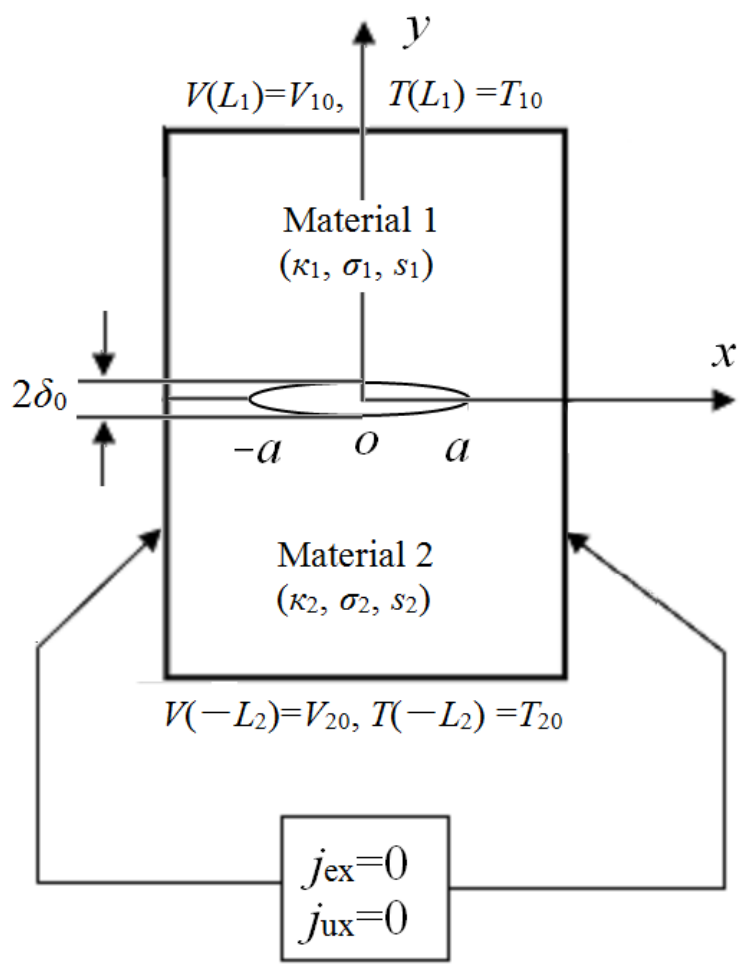

Fig. 1 


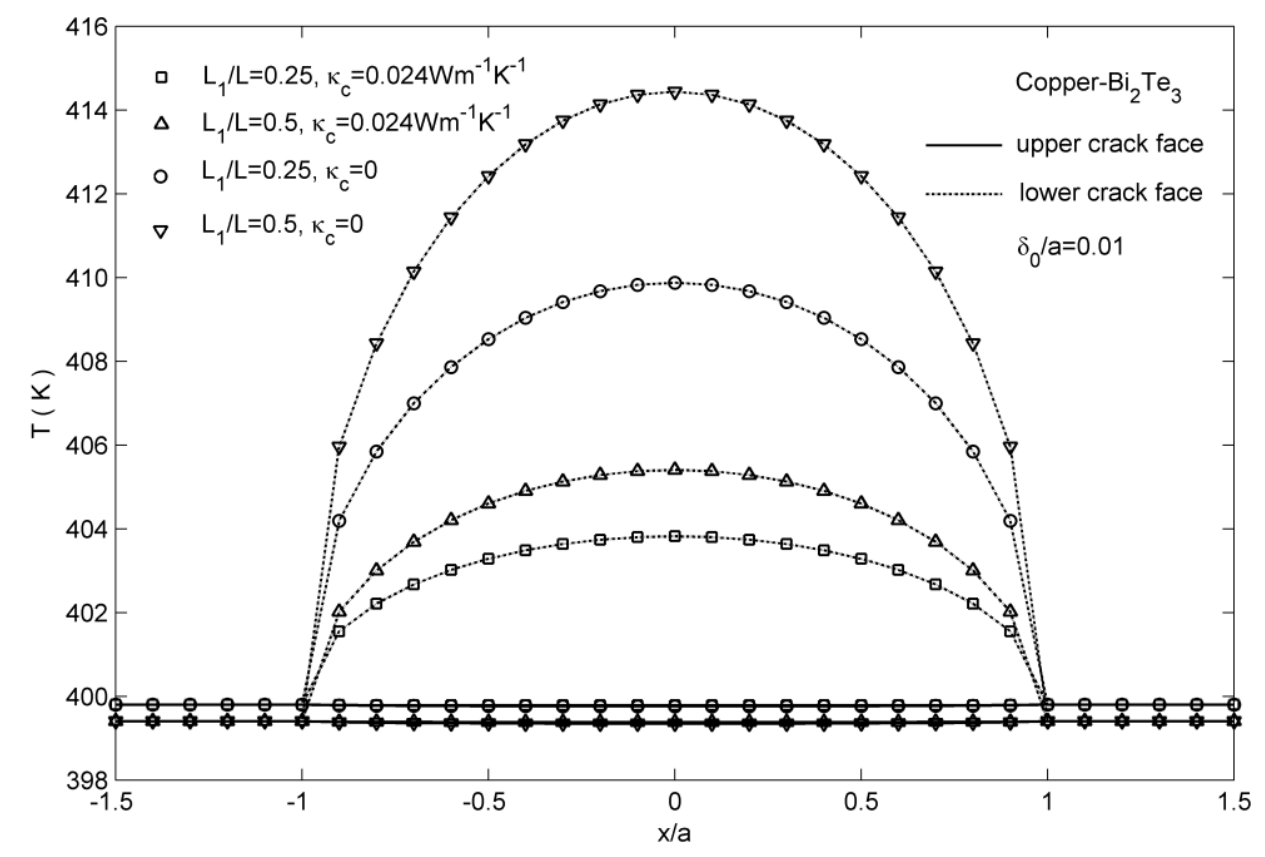

Fig. 2 


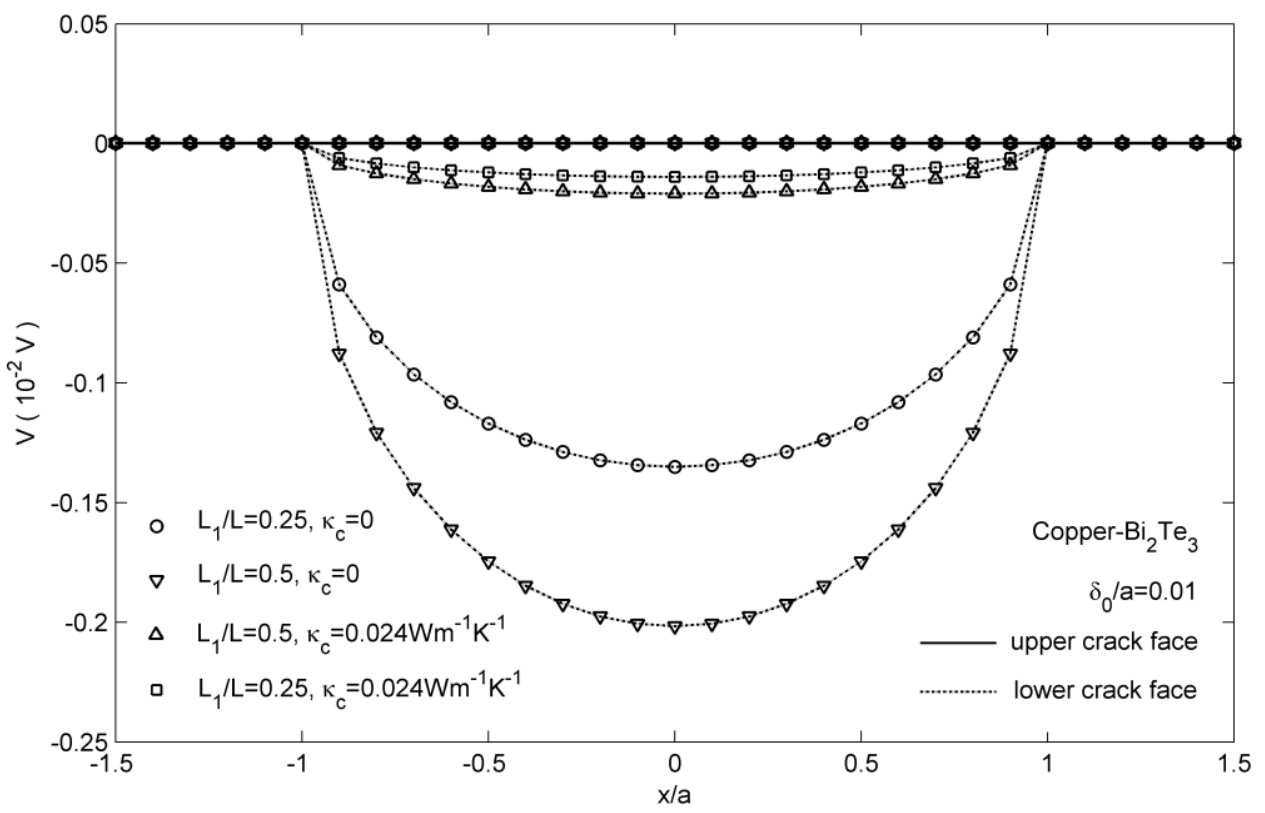

Fig. 3 


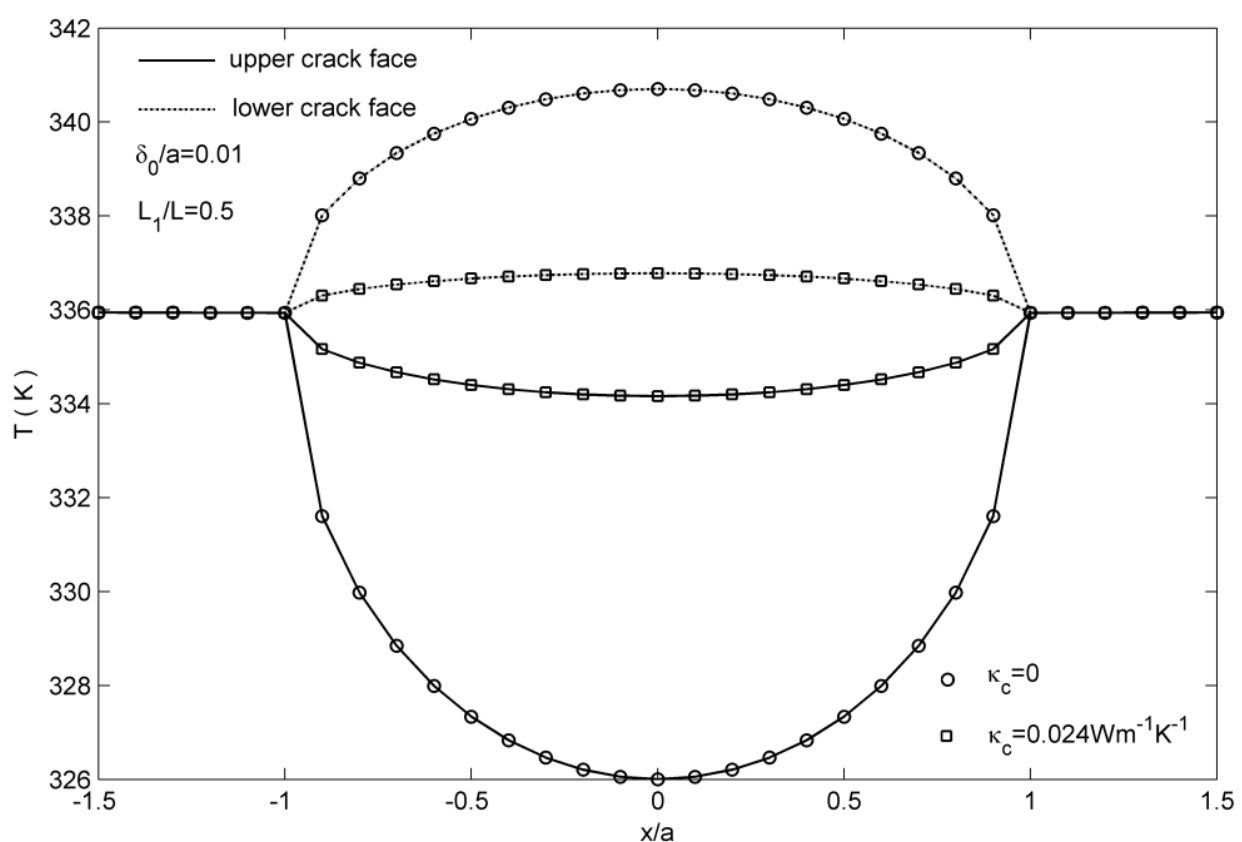

Fig. 4 


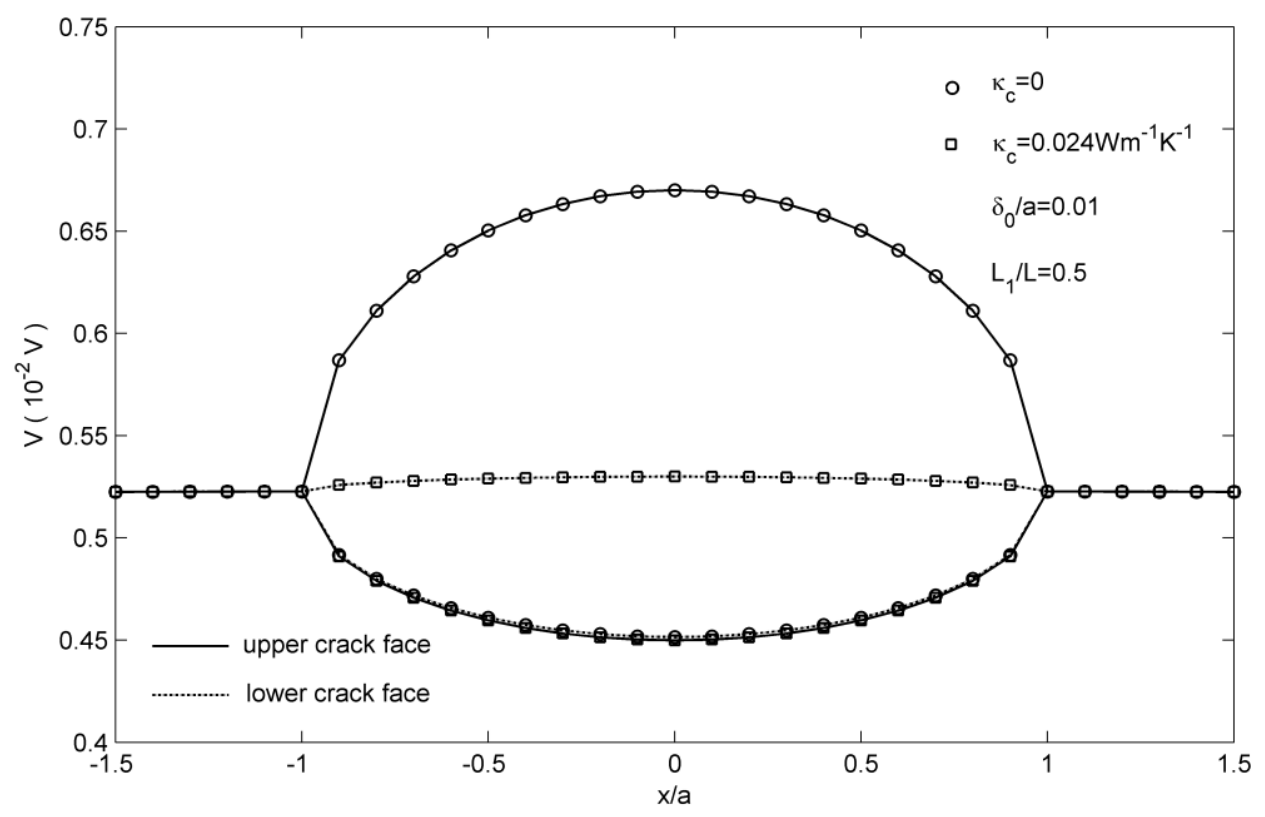

Fig. 5 


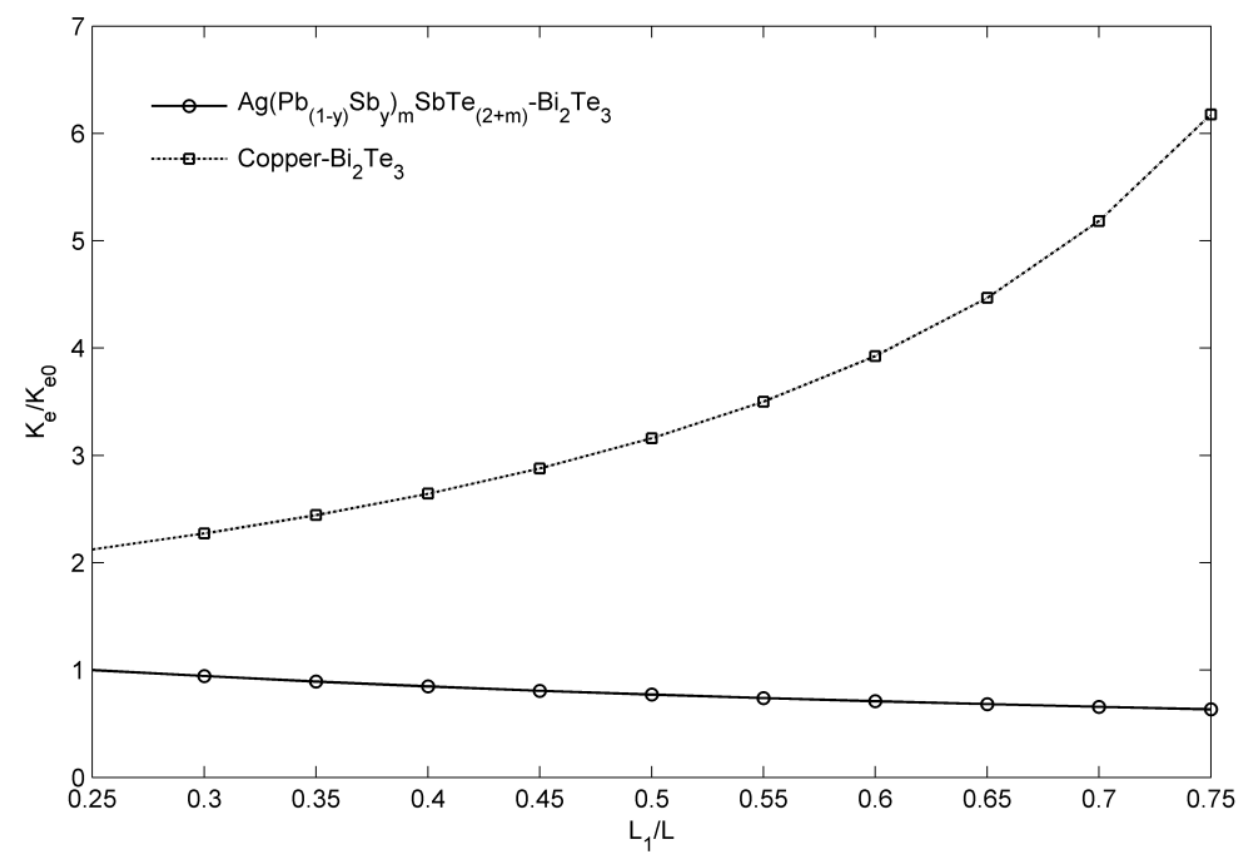

Fig. 6 


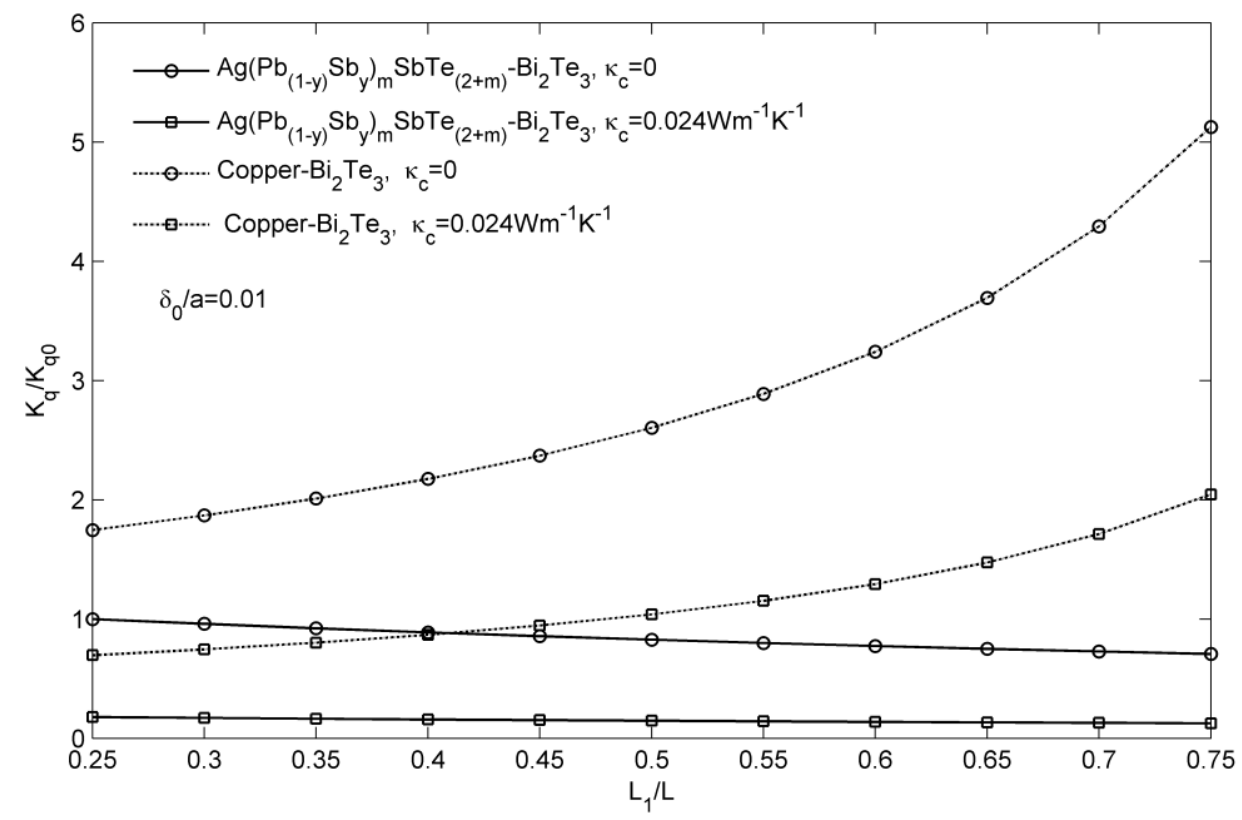

Fig. 7 


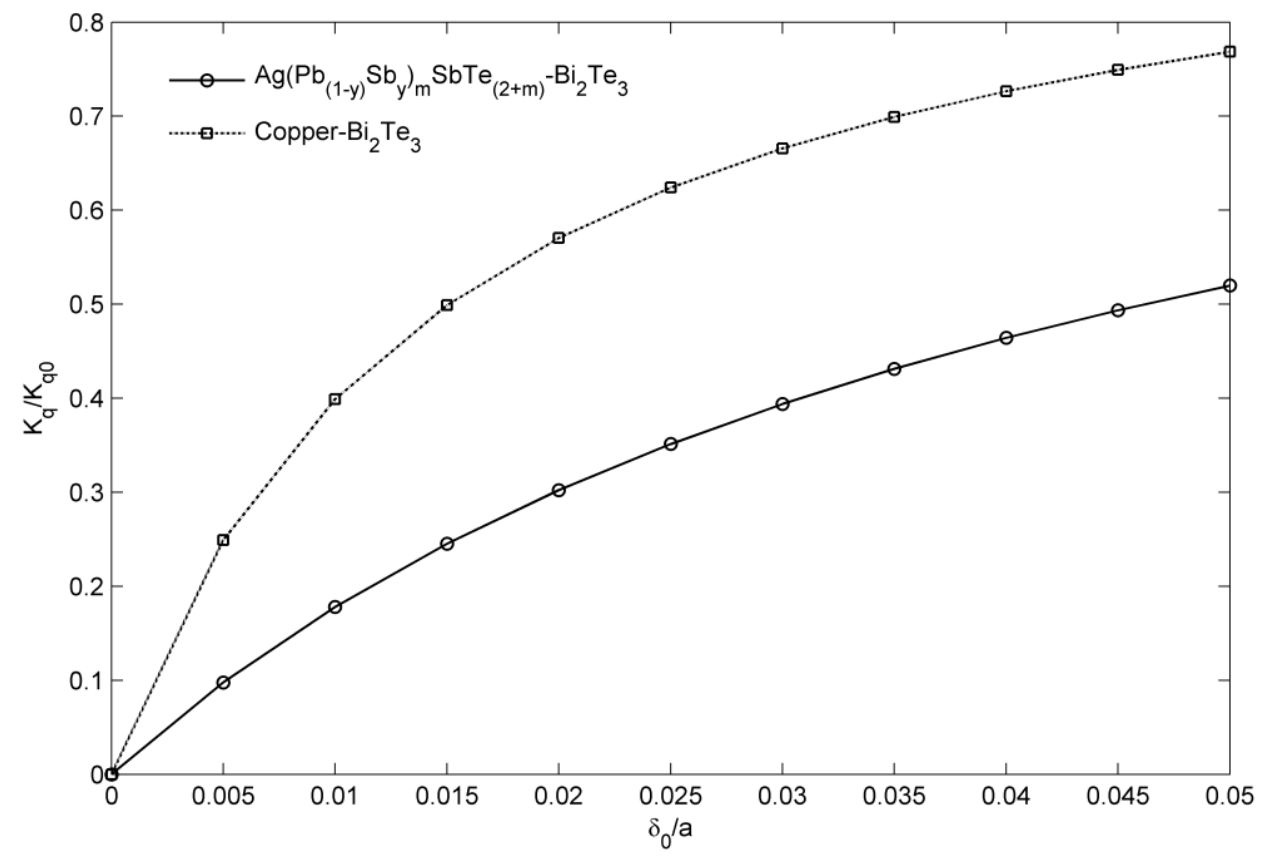

Fig. 8 
Table 1 Material properties

\begin{tabular}{cccc}
\hline \multirow{2}{*}{ Material } & Heat conductivity & $\begin{array}{c}\text { Electric } \\
\text { conductivity }\end{array}$ & Seebeck \\
& $\kappa\left(\mathrm{Wm}^{-1} \mathrm{~K}^{-1}\right)$ & $\sigma\left(\mathrm{Sm}^{-1}\right)$ & $s\left(\mathrm{VK}^{-1}\right)$ \\
\cline { 2 - 4 } & 0.77 & $2.2 \times 10^{4}$ & $2.7 \times 10^{-4}$ \\
$\mathrm{Ag}\left(\mathrm{Pb}_{1-\mathrm{y}} \mathrm{Sn}_{\mathrm{y}}\right)_{\mathrm{m}} \mathrm{SbTe}_{2+\mathrm{m}}$ & 1.6 & $1.1 \times 10^{5}$ & $2.0 \times 10^{-4}$ \\
$\mathrm{Bi}_{2} \mathrm{Te}_{3}$ & 400 & $5.88 \times 10^{8}$ & $6.5 \times 10^{-6}$ \\
$\mathrm{Copper}_{\text {Air }}$ & 0.024 & - & - \\
\hline
\end{tabular}

